\title{
Am Puls der Hausärzte
}

Erhard Taverna

\section{«200 Stunden Filmmaterial, denen ein Casting}

mit eintägigen Probeaufnahmen in dreissig Praxen vorausging.»

Sylviane Gindrat, Ärztin in Bern, Ethnologin und Regisseurin, hat in ihrer Dokumentarfilm-Trilogie vier Hausärzte und zwei Hausärztinnen in der Praxis filmisch begleitet. Die Filme wurden in mehreren Kinos und an Kongressen gezeigt. Im Februar 2014 ist erstmals eine Ausstrahlung über das Fernsehen geplant.

«Das Wesen der Hausarztmedizin kann nur durch eine direkte Beobachtung in der Sprechstunde erfasst werden, dort, wo üblicherweise kein Aussenstehender Zugang hat», sagt die Filmemacherin, die selber seit 20 Jahren eine Einzelpraxis in Bern betreibt. Die Idee, diesen Kern hausärztlichen Wirkens filmisch darzustellen, sei ihr 2006 in ihrem Qualitätszirkel gekommen, wo Komplexität und Qualitätskriterien ausdiskutiert wurden. Das Interesse an der narrativen Medizin und dem sozio-kulturellen Umfeld der Betroffenen ergaben das Leitmotiv für die aufwendigen Dreharbeiten in fünf verschiedenen Praxen. Eine ideale Voraussetzung für 200 Stunden Filmmaterial, denen ein Casting mit eintägigen Probeaufnahmen in dreissig Praxen vorausging. Aus-

wahlkriterien waren Vielfalt, wie Beispiele aus der Romandie und Deutschschweiz, Stadt und Land, von Jung und Alt, Mann und Frau. Der erste Teil erzählt von einem Ärzte-Ehepaar mit vier Kindern, Stéphane und Franziska, das die Grundversorgung im Val d'Anniviers sicherstellt. Das Spektrum reicht von der Inneren Medizin, Pädiatrie und Traumatologie bis zur Bergrettung. Im zweiten Teil kommen Gabi und Bruno zu Wort. Sie, die begeisterte Ärztin,

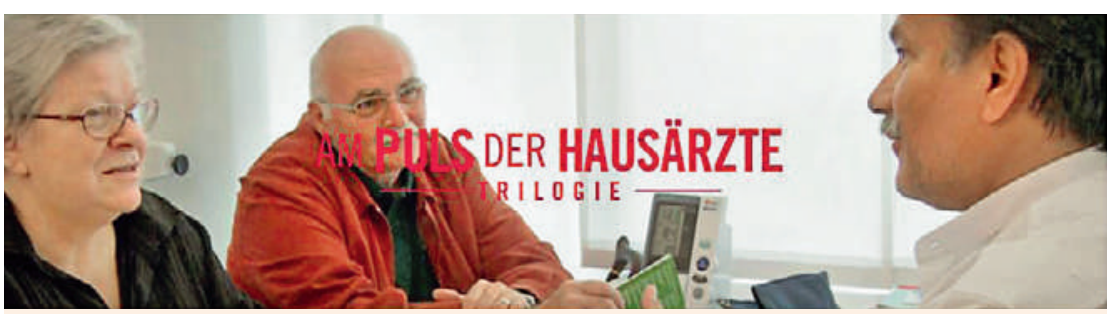

Für Sylviane Gindrat war es fast nie ein Problem, die Zustimmung der Patienten für die Filmaufnahmen zu gewinnen.

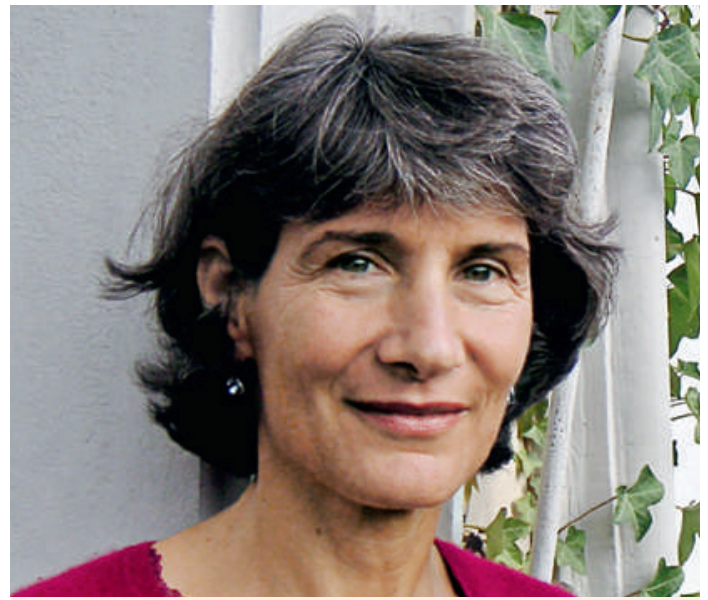

Die Hausärztin und Filmemacherin Sylviane Gindrat: «Das Wesen der Hausarztmedizin kann nur durch direkte Beobachtung in der Sprechstunde erfasst werden.»

noch in der Weiterbildung, er, der erfahrene Hausarzt in einem Berner Quartier, vielseitig engagiert als Lehrer und Publizist. Der dritte Teil zeigt zwei Generationen von Hausärzten. Da ist Sébastien, der in einer Lausanner Gruppenpraxis arbeitet, Notfälle im Ambulatorium betreut und im Rahmen des «Cursus romand de médecine de famille» Laufbahnberatungen für junge Ärzte mit Interesse an Hausarztmedizin anbietet. Ihm gegenüber steht Paul im Entlebuch, heute im Pensionsalter, ein engagierter, unermüdlicher Schaffer auf dem Lande, der keinen Nachfolger findet.

Für sie als Ärztin sei es kein Problem gewesen, die Zustimmung fast aller Patient(inn)en zu gewinnen, die sie jeweils erst kurz vor den Aufnahmen im Wartezimmer informierte. Keine Einstellung sei wiederholt worden. Oft hätten die Akteure die Anwesenheit der Regisseurin und ihres Teams (zweite Kamera und ein Tonmeister) einfach vergessen. Für die Filmemacherin, beobachtende Ethnologin und Ärztin hätten Innen- und Aussensicht ständig gewechselt.

Die Trilogie ist von Anfang an eine Erfolgsgeschichte. Begeistert am Welt-Hausärztekongress 2013 in Prag aufgenommen, von Studenten gefeiert und für Lehrzwecke von medizinischen Fakultäten aller Kontinente angefragt. Einzelne Filmsequenzen sollen kommentiert im Unterricht verwendet werden. Es folgen Einladungen zu Workshops, weiteren Kongressen, Eröffnung neuer Studienfächer und Rundtischgesprächen. Die Publizität verdankt sich der exakten, kommentarlosen und einfühlsamen Beobachtung, die enorm authentisch wirkt. Der Film 


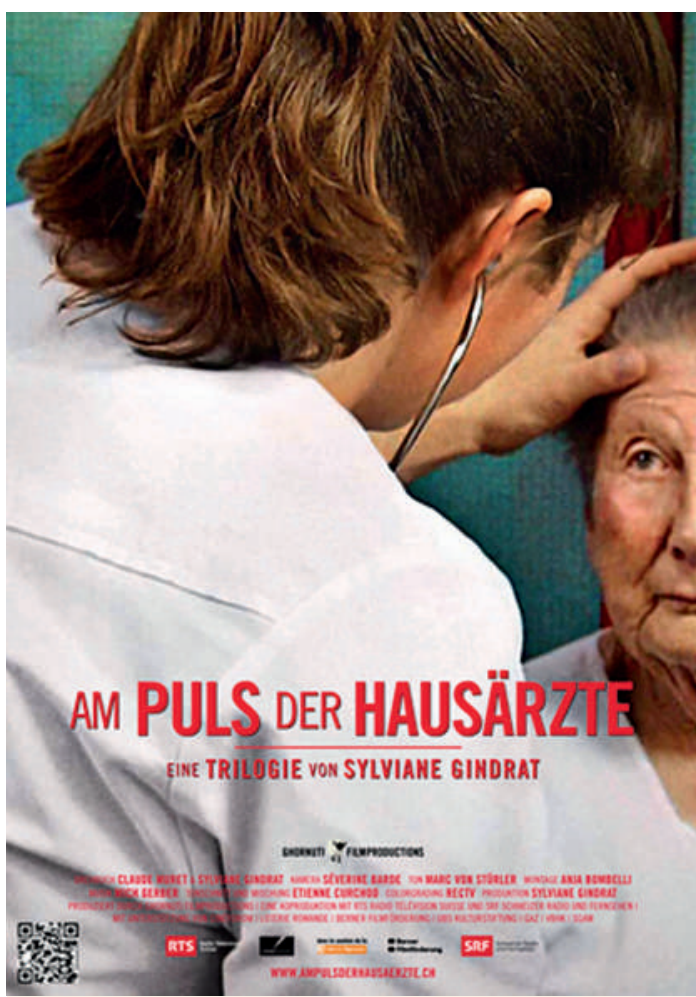

«Am Puls der Hausärzte», eine Film-Trilogie von Sylviane Gindrat, TV-Ausstrahlung im Februar 2014. DVD erhältlich ab März 2014 bei info[at]ghornuti.ch www.ampulsderhausaerzte.ch wenn sie nicht gerade auf Promotionstour ist oder einer neuen Einladung folgt.

Ihre Energie bezieht Sylviane Gindrat aus der lebenslänglichen Neugier an menschlichen Lebenskontexten. Das war auch der Grund, nach zehn Praxisjahren 1998 berufsbegleitend ein Zweitstudium in Sozialanthropologie und Filmwissenschaften in Bern und Zürich zu beginnen. Für sie, als visuell orientierte Person, ist der Film ein ausgezeichnetes Medium, gesellschaftliche Themen darzustellen. Ein Glücksfall für die Hausarztmedizin, eine gewichtige Verstärkung aller Kreise, die sich seit Jahren um eine Besserstellung der Hausarztmedizin bemühen.

Die Trilogie hat vielleicht das Potential, den politischen Richtungskämpfen einen neuen Weg zu weisen. Sylviane Gindrat ist überzeugt, dass der Beziehung in der Therapie eine wichtige Bedeutung zukommt. Gerade auch wegen der fortgeschrittenen Technisierung und sozialmedizinischen Fragmentierung der modernen Medizin. Der Film idealisiert nicht, er zeigt, was bei den dargestellten sechs Hausärzten der Fall ist. Daraus ergeben sich Fragen, aber keine Lösungen. Letztere bleiben Sache der Politik und der Gesellschaft. Was als Eindruck auch bleibt, ist die enorme Vielfalt möglicher Praxisarbeit. Auch das lässt Fragen offen, nach dem idealen Curriculum oder nach zukünftigen Arbeitsformen. Jenseits der seriellen TV-Fiktionen vom Land- und Bergarzt sind offensichtlich Dokufilme machbar, die auch ohne Sex and Crime spannende Unterhaltung bieten. Der

\section{Gesundheitspolitiker hätten versichert, dass ihnen erstmals klar geworden sei, was Hausarztmedizin bedeuten könne.}

weckt Emotionen, wer ähnliche Berufserfahrungen hat, identifiziert sich sofort mit den gezeigten Darstellern. Es sei vorgekommen, dass gestandene Kollegen zu Tränen gerührt waren, und ausserdem hätten Gesundheitspolitiker versichert, dass ihnen erstmals klar geworden sei, was Hausarztmedizin bedeuten könne.

Sylviane Gindrat hat schon mit dem Erstling «Destination Liebe» und der folgenden Doku «Schneeweisse Schwarznasen» über Walliser Schafzüchter Filmpreise gewonnen. Eine Filmproduktion benötigt Finanzen und Mitarbeiter. Zur Bewältigung aller damit verbundenen Aufgaben hat sie ihre eigene Produktionsfirma «Ghornuti Filmproductions» mit Sitz im französischsprachigen Wallis gegründet. Sie pendelt zwischen Bern und Ayent hin und her,
Ärztin und Filmemacherin ist, nicht ohne Eigennutz, in beiden Berufen weiterhin viel Erfolg zu wünschen, denn einen solchen Katalysator hat man sich schon lange gewünscht.

\section{Fernsehausstrahlungen}

SRF: 19.2.2013 «Am Puls der Hausärzte» (Stéphane \& Franziska, Gabi \& Bruno, Paul \& Sébastien)

RTS: 19.2.2014 «Du côté des médecins» (Stéphane \& Franziska); 21.2.2014 (Gabi \& Bruno); 24.2.2014 (Paul \& Sébastien)

\section{RSI: März 2014}

Aktuelle Informationen zu Kino, TV und DVD: www.ampulsderhausaerzte.ch 